\title{
Effect of swallowing maneuver on fentanyl-induced coughing
}

\author{
Chun-Ning Ho ${ }^{1}$ Kuo-Chuan Hung ${ }^{1}[$
}

Received: 8 June 2017 / Accepted: 6 July 2017 / Published online: 18 July 2017

(C) Japanese Society of Anesthesiologists 2017

Keywords Opioid $\cdot$ Cough $\cdot$ Fentanyl $\cdot$ Swallowing

To the Editor:

We read with interest the recent article by Sako et al. [1] that described a swallowing maneuver that reduces the incidence of fentanyl-induced coughing (FIC) (from 40.4 to $14.0 \%$ ) when performed more than five times immediately before intravenous fentanyl administration [1]. Undoubtedly, Sako et al. [1] have proposed a simple yet effective method to suppress FIC. Although they provided a possible explanation for their finding, the mechanism underlying FIC suppression by the swallowing maneuver was not well clarified. We wish to provide a more detailed discussion regarding the suppressive effect of the swallowing maneuver on FIC.

By investigating the association between swallowing reflex and airway defense in an animal model, Tsujimura et al. found that the swallowing reflex can be evoked by airway stimulation [2]. Furthermore, it was probably induced by the activation of the same afferent nerves that regulate the cough reflex, but occurred at lower stimulus intensities. The authors suggested that the laryngeal and tracheal afferent nerves that regulate swallowing and cough reflexes are the same [2]. They also found that nerves regulating the cough reflex can become desensitized when evoked

This comment refers to the article available at doi:10.1007/ s00540-016-2300-4.

Kuo-Chuan Hung

ed102605@gmail.com

1 Department of Anesthesiology, Chi Mei Medical

Center, No. 901, Chung Hwa Road, Yung Kung Dist.,

Tainan County 710, Taiwan, ROC by repetitive electrical stimulation of the tracheal mucosa. In the study by Sako et al. [1], there is a possibility that the afferent nerves that regulate the cough reflex become desensitized after repeated swallowing maneuvers, which may explain its suppressive effect on FIC.

Compliance with ethical standards

Conflict of interest No competing interests declared.

\section{References}

1. Sako S, Tokunaga S, Tsukamoto M, Yoshino J, Fujimura N, Yokoyama T. Swallowing action immediately before intravenous fentanyl at induction of anesthesia prevents fentanylinduced coughing: a randomized controlled study. J Anesth. 2017;31:212-8.

2. Tsujimura T, Udemgba C, Inoue M, Canning BJ. Laryngeal and tracheal afferent nerve stimulation evokes swallowing in anaesthetized guinea pigs. J Physiol. 2013;591:4667-79. 